\title{
Vigilância em saúde do trabalhador: o suicídio relacionado ao trabalho*
}

\author{
Carlos Alexandre Curylofo Corsi ${ }^{1,2}$ \\ (D) https://orcid.org/0000-0002-2983-1436 \\ Alan Vinicius Assunção Luiz ${ }^{3,4}$ \\ (1) https://orcid.org/0000-0003-3644-3981 \\ Álefe Saloum Cintra ${ }^{3}$ \\ (D) https://orcid.org/0000-0002-7555-5524 \\ Natássia Condilo Pitta ${ }^{3,4}$ \\ (D) https://orcid.org/0000-0003-0098-3310 \\ Ana Cláudia da Silva Paschoal ${ }^{5}$ \\ (D) https://orcid.org/0000-0003-3664-4863 \\ Talita Silva Queiroz ${ }^{5}$ \\ (D) https://orcid.org/0000-0002-3416-8843 \\ Milena Flória-Santos 3,4 \\ (DD https://orcid.org/0000-0002-0656-1736
}

Objetivo: investigar as possíveis relações que permeiam as temáticas da ideação suicida e do suicídio associadas aos aspectos internos e externos do trabalho. Método: ao adotar uma abordagem qualitativa e perspectiva descritiva, foi realizada uma revisão integrativa da literatura do período de 2009-2019 operacionalizada por buscas nas bases de dados PubMed, SCOPUS, Web of Science, LILACS, BDENF e BVS. Resultados: partiu-se da questão norteadora "Quais são as relações existentes entre o trabalhador, vítima de suicídio, e o ambiente de trabalho em que ele está inserido?" e de descritores, encontrando-se 481 registros, dos quais dez estudos foram selecionados. Separados por dois eixos de análise (aspectos internos e externos), os estudos descreveram dez relações predominantes entre o suicídio e o trabalho, sendo elas: depressão (19,3\%); assédio moral/bullying $(16,1 \%)$; ausência de lazer (12,9\%); estresse (9,6\%); sobrecarga profissional $(9,6 \%)$; acidentes no trabalho (9,6\%); Síndrome de Burnout $(6,4 \%)$; isolamento social $(6,4 \%)$; conflitos entre a família e o trabalho $(6,4 \%)$ e falta de autonomia no trabalho $(3,2 \%)$. Conclusão: os resultados demonstram a relação existente entre o trabalho e o suicídio. Como a Enfermagem é uma potencial promotora no cuidado humanizado, destacase a necessidade de vigilância e promoção da saúde para os trabalhadores nesses ambientes.

Descritores: Suicídio; Saúde do Trabalhador; Vigilância em Saúde Pública; Riscos Ocupacionais.

\section{Como citar este artigo}

Corsi CAC, Assunção-Luiz AV, Cintra AS, Pitta NC, Paschoal ACS, Queiroz TS et al. Worker's health surveillance: work-related suicide. SMAD, Rev Eletrônica Saúde Mental Álcool Drog. 2020;16(4):133-143. doi: https://dx.doi.org/10.11606/issn.1806-6976.smad.2020.172196 


\section{Worker's health surveillance: work-related suicide}

Objective: to investigate the possible links between the themes of suicide ideation and suicide associated with the internal and external aspects of the work. Method: by adopting a qualitative approach and descriptive perspective, an integrative review of the literature of the 2009-2019 period was carried out by searching the PubMed, SCOPUS, Web of Science, LILACS, BDENF and VHL databases. Results: The guiding question "What are the relations between the worker, victim of suicide, and the work environment in which he is inserted?" and descriptors, with 481 records, of which ten studies, were selected. Separated by two axes of analysis (internal and external aspects), the studies described ten predominant relationships between suicide and work, being: depression (19.3\%); moral harassment/bullying (16.1\%); absence of leisure (12.9\%); stress $(9.6 \%)$; professional overload (9.6\%); accidents at work (9.6\%); Burnout syndrome (6.4\%); social isolation (6.4\%); conflicts between family and work (6.4\%) and lack of autonomy at work $(3.2 \%)$. Conclusion: the results show the relationship between work and suicide. As nursing is a potential promoter of humanized care, the need for vigilance and health promotion for workers in these environments is highlighted.

Descriptors: Suicide; Worker's Health; Public Health Surveillance; Occupational Risks.

\section{Vigilancia de la salud del trabajador: suicidio relacionado con el trabajo}

Objetivo: investigar las posibles relaciones que impregnan los temas de ideación suicida y suicidio, asociados con aspectos internos y externos del trabajo. Método: adoptando un enfoque cualitativo y una perspectiva descriptiva, se realizó una revisión integradora de la literatura para el período 2009-2019 mediante la búsqueda en las bases de datos PubMed, SCOPUS, Web of Science, LILACS, BDENF y BVS. Resultados: Basado en la pregunta guía: "¿Cuáles son las relaciones que existen entre el trabajador, una víctima de suicidio y el ambiente de trabajo en el que está insertado?" y descriptores, se encontraron 481 registros, de los cuales se seleccionaron 10 estudios. Separados por dos ejes de análisis (aspectos internos y externos), los estudios describieron 10 relaciones predominantes entre el suicidio y el trabajo, a saber: depresión $(19.3 \%)$, bullying / bullying $(16.1 \%)$, ausencia de ocio $(12.9 \%)$, estrés $(9.6 \%)$, sobrecarga profesional $(9.6 \%)$, accidentes laborales $(9.6 \%)$, síndrome de Burnout $(6.4 \%)$, aislamiento social $(6,4 \%)$, conflictos entre familia y trabajo $(6,4 \%)$ y falta de autonomía en el trabajo $(3,2 \%)$. Conclusión: los resultados demuestran una relación entre el trabajo y el suicidio. Como la enfermería es un potencial promotor de la atención humanizada, se destaca la necesidad de vigilancia y promoción de la salud de los trabajadores en estos entornos.

Descriptores: Suicidio; Salud Laboral; Vigilancia en Salud Pública; Riesgos Laborales. 


\section{Introdução}

O suicídio é classificado como violência autoinfligida relacionada ao comportamento suicida e às diversas formas de automutilação, que vão desde pensamentos autodestrutivos, elaboração de planos para se ferir (pensamento suicida) até a realização do ato de autoextermínio para acabar com a própria vida(1).

Segundo a Organização Mundial da Saúde (OMS), o suicídio é atualmente um problema de saúde pública mundial(1). A tentativa prévia é o fator de risco mais importante para o suicídio na população em geral e a taxa de mortalidade por suicídio no mundo é de $57 \%$, superando a mortalidade causada por guerras ou homicídios ${ }^{(1-2)}$. Diante da situação supracitada, recomenda-se que o suicídio seja discutido e priorizado nas agendas de saúde a fim de suscitar a formulação de políticas públicas que atendam aos principais grupos de risco $^{(2-3)}$.

Fatores estressores internos e externos de um determinado ambiente podem ser considerados gatilhos para o empobrecimento de saúde mental. Assim sendo, o ambiente de trabalho também pode ser considerado um fator promotor de saúde mental, já que o mesmo proporciona interações sociais do indivíduo(4-5).

As interações internas e externas do indivíduo nos ambientes trabalhistas promovem autoestima, resiliência e habilidades emocionais, porém, se comparadas a ambientes estressores, podem propiciar sofrimento, adoecimento e pensamentos suicidas. Mediante a isso, a OMS determinou, em 2019, a priorização de notificação para as doenças relacionadas ao trabalho(1,5).

Situações negativas recorrentes no cenário do trabalhador são fatores de inclusão para estressores mentais, que podem influenciar o pensamento suicida e o suicídio, os quais se destacam, por exemplo: assédio ou bullying; depressão; Síndrome de Burnout; condições do ambiente de trabalho; excesso de horas, entre outros ${ }^{(6)}$. Assim, em uma perspectiva mais ampla, relacionada à saúde do trabalhador, ao ambiente de trabalho e consecutivamente baseada nas muitas maneiras pelas quais o suicídio pode estar associado, é possível que haja uma intrínseca relação entre essas áreas de estudo(3).

A produção científica referente aos transtornos relacionados ao trabalho ainda é incipiente. Assuntos que abordam esses temas são pouco discutidos nas esferas públicas de saúde, gerando a falta de incentivos de promoção à saúde desse público, muitas vezes negligenciado nas ações dos Centros de Referência Especializados em Saúde do Trabalhador (CEREST) e da Rede Nacional de Atenção à Saúde dos Trabalhadores (RENAST), que integram os serviços do Sistema Único de Saúde (SUS) $)^{(7-8)}$.
A Política Nacional de Saúde do Trabalhador e da Trabalhadora (PNSTT), junto à Política Nacional de Segurança e Saúde no Trabalho (PNSST), tem por objetivos: a promoção da saúde; a melhoria da qualidade de vida; a prevenção de acidentes e de danos à saúde advindos e/ou relacionados ao trabalho. Seus escopos dão-se para o desenvolvimento da atenção integral à saúde do trabalhador junto ao Sistema Único de Saúde (SUS), com ênfase na vigilância e redução da morbimortalidade dos trabalhadores decorrentes dos modelos de desenvolvimento e dos processos produtivos $^{(7-9)}$

Assim, no âmbito da vigilância em saúde do trabalhador, esse olhar prévio, mais criterioso, facilitaria a promoção da saúde, buscando identificar estratégias mais adequadas e oportunas, evitando, assim, casos extremos, como, por exemplo, o autoexterminínio(10).

Portanto, este estudo propõe-se à investigação de possíveis relações que permeiam as temáticas da ideação suicida e do suicídio, associadas aos aspectos internos e externos do trabalho, nos últimos dez anos, por meio de uma Revisão Integrativa da Literatura Científica (RI), visando, assim, a suscitar ações de vigilância em saúde no trabalho e reconhecimento desses trabalhadores, prevenindo danos mais graves.

\section{Método}

Estudos de RI possibilitam, ao pesquisador, a inclusão de estudos experimentais e não experimentais, sintetizando múltiplos trabalhos publicados para conclusões abrangentes sobre determinada área de estudo. Isso permite a combinação de dados teóricos e empíricos da literatura, auxiliando na compreensão do fenômeno analisado e identificando eventuais lacunas de investigações para pesquisas futuras ${ }^{(11-13)}$.

Neste estudo, foram seguidas as seis etapas propostas para a confecção da $\mathrm{RI}^{(14)}$, sendo elas: formulação da pergunta norteadora; amostragem ou busca na literatura dos estudos; extração dos resultados; análise crítica; análise e síntese dos resultados da revisão; apresentação da RI.

Considerada como padrão-ouro da pesquisa científica, a Prática Baseada em Evidência (PBE) é hoje utilizada como um norteador para classificar a qualidade dos estudos científicos, realizados principalmente nas áreas da saúde, indo ao encontro de necessidades práticas de detecção para a melhoria do cuidado humanizado de equipes multiprofissionais e principalmente da equipe de Enfermagem(11-12).

Portanto, a definição da questão norteadora da pesquisa foi realizada pela estratégia $\mathrm{PICo}^{(15)}$, o que proporcionou, aos pesquisadores, condições para as buscas. Tal pergunta orientara os critérios de elegibilidade expressos onde: $\mathrm{P}=$ participante, $\mathrm{I}=$ 
intervenção e Co $=$ contexto/resultados ${ }^{(15)}$. Para este estudo, respectivamente: População = trabalhadores: indivíduos que ocupam determinados ambientes de trabalho, não especificados; Intervenção = suicídio: indivíduos vítimas de suicídio com possível relação com o trabalho; Contexto/resultados = vigilância em saúde do trabalhador: características do processo e/ou ambiente e/ou relações de trabalho.

Assim, com base em tal estratégia metodológica, a questão norteadora desta revisão foi construída da seguinte maneira: "Quais são as relações existentes entre o trabalhador, vítima de suicídio, e o ambiente de trabalho em que ele está inserido?".

Diante da emergente discussão sobre a temática, optou-se por um recorte amostral dos últimos dez anos (2009-2019), tendo em vista que discussões sobre o suicídio associado ao trabalho são extremamente recentes. Além dos artigos originais, dissertações e teses publicadas no período foram incluídas, nos idiomas português, inglês e espanhol, assim como estudos de abordagem quantitativa, qualitativa e relatos de experiência.

Foram excluídos artigos de revisão da literatura, protocolos, cartas, editoriais e artigos que trouxeram a temática do suicídio no espaço físico do trabalho onde o indivíduo tenha cometido o suicídio.

A escolha dos descritores foi feita por meio de buscas, consultando os Descritores em Ciências da Saúde (DeCS) controlados na Biblioteca Virtual de Saúde (BVS) e os Medical Subject Headings (MeSH) via National Center for Biotechnology Information (NCBI). Descritores controlados são aqueles utilizados para indexação e busca de artigos nas bases de dados sobre determinado tema e os descritores não controlados representam palavras textuais e seus sinônimos(12-13) (Figura 1).

\begin{tabular}{|c|c|c|}
\hline \multicolumn{3}{|c|}{ Descritores controlados } \\
\hline & DeCS & $\mathrm{MeSH}$ \\
\hline$P$ & $\begin{array}{l}\text { Trabalhadores } \\
\text { Saúde do trabalhador }\end{array}$ & $\begin{array}{l}\text { Occupational Groups } \\
\text { Work } \\
\text { Workforce } \\
\end{array}$ \\
\hline I & $\begin{array}{l}\text { Suicídio } \\
\text { Tentativa de suicídio } \\
\text { Suicídio assistido } \\
\text { Ideação suicida } \\
\text { Satisfação no emprego } \\
\text { Mortalidade ocupacional } \\
\text { Equilíbrio trabalho-vida }\end{array}$ & $\begin{array}{l}\text { Suicide, Attempted } \\
\text { Suicide } \\
\text { Suicide, Assisted } \\
\text { Suicidal Ideation } \\
\text { Job Satisfaction } \\
\text { Work-Life Balance }\end{array}$ \\
\hline Co & $\begin{array}{l}\text { Vigilância em saúde pública } \\
\text { Ambiente de trabalho } \\
\text { Local de trabalho } \\
\text { Riscos Ocupacionais } \\
\text { Emprego } \\
\text { Condições de trabalho }\end{array}$ & $\begin{array}{l}\text { Employment } \\
\text { Workplace }\end{array}$ \\
\hline
\end{tabular}

Figura 1 - Descritores controlados e não controlados utilizados na pesquisa. Ribeirão Preto, SP, Brasil, 2019

Os termos apresentados na Figura 1 são conectores determinantes para a realização das buscas, permitindo a realização de combinações. As variações no uso de operadores booleanos (and, or, and not) favorecem a proximidade nas frases de estratégia de busca formadas. Desse modo, utilizaram-se diferentes combinações entre os descritores, palavras-chave e booleanos(13-14).

As buscas foram realizadas no portal PubMed e nas seguintes bases de dados: SCOPUS, Web of Science, LILACS e BDENF. O critério dessas escolhas deu-se por meio da notoriedade de indexação de cada base de dados, contendo arsenal de títulos sobre assuntos de interesse à temática, abrangendo as grandes áreas de concentração ligadas à saúde, voltadas principalmente às temáticas: Enfermagem; Psicologia; saúde do trabalhador; vigilância em saúde pública e Medicina, atendendo, assim, ao propósito de representar um índice bibliográfico composto por referências da literatura técnico-científica nacionais e internacionais ${ }^{(12)}$.

Para cada base de dado escolhida para a revisão, foram identificadas as melhores estratégias de busca, levando em consideração a especificidade de suas interfaces $^{(12,15)}$. Desde a apresentação e sequência no rigor das buscas, até a divulgação desses resultados, optou-se em utilizar o fluxograma PRISMA ${ }^{(16-17)}$.

Com o trabalho de dois revisores, que operacionalizaram as buscas de forma independente desde a identificação dos artigos, durante a etapa de seleção inicial do processo, até a amostra final na etapa final de inclusão, foi possível obter os estudos potencialmente elegíveis para compor a RI.

Todas as buscas foram idealizadas e organizadas previamente, sendo realizadas em único dia no mês de novembro de 2019. Convergências e divergências entre os dois pesquisadores foram submetidas a um terceiro pesquisador, que definiu, como pertinente ao foco da RI, o conteúdo identificado nos artigos.

\section{Resultados e discussão}

Após a operacionalização no portal e bases de dados e exclusão dos artigos duplicados, 481 trabalhos foram identificados nas buscas, iniciando-se o processo de seleção. Desses, um estudo foi excluído por limite temporal e 480 foram eleitos para a leitura do resumo. Nessa etapa de seleção, procedeu-se à leitura dos resumos selecionados, sendo excluídos 455 trabalhos e selecionados 25 estudos para a leitura na íntegra. Na etapa de elegibilidade, fez-se a leitura na íntegra dos trabalhos selecionados, sendo excluídos 15 estudos. Na etapa final de inclusão, dez estudos foram enquadrados nesta RI, de acordo com os critérios de inclusão e exclusão apresentados. O processo de identificação, seleção, elegibilidade e inclusão estão descritos por meio do fluxograma PRISMA ${ }^{(17)}$ 
(Figura 2). Após a seleção, para melhor visualização e discussão dos resultados, elaborou-se uma tabela com as características básicas dos estudos encontrados (Figura 3).



Figura 2 - Fluxograma com o resultado das buscas nas bases de dados e no portal PubMed. Ribeirão Preto, SP, Brasil, 2009-2019

A partir dos dez estudos eleitos com base nos critérios delimitados para esta RI, constatou-se que houve predominância de estudos qualitativos entre os artigos localizados (90\%), com a ocorrência de apenas um único artigo quantitativo (10\%). Os estudos são oriundos de sete países diferentes, dos quais se destaca a Austrália com a maioria dos artigos publicados (30\%) e consecutivamente: Japão (20\%); China (10\%); Estados Unidos (10\%); Alemanha (10\%); Reino Unido (10\%) e Brasil (10\%). Em relação à área de concentração do primeiro autor, contribuíram para os estudos: Medicina (40\%) e Psicologia (30\%), seguidas de Medicina Veterinária (20\%) e Administração (10\%). Para a elaboração dos estudos, os autores utilizaram predominantemente bancos de dados (60\%), questionários (30\%) e entrevista $(10 \%)$, consecutivamente, sendo eles aplicados em grupos mistos de pessoas, homens e mulheres, com amostragem maior para o público masculino. Em relação à quantidade de publicações sobre o tema localizadas cronologicamente na RI, a primeira contribuição deu-se em 2009 e a última, em 2017, sendo esse o ano com maior número de publicações (30\%).

Empregou-se, considerando-se os assuntos abordados nos resultados dos estudos, a técnica de
Análise de Conteúdo para estruturar categorias temáticas a partir do agrupamento de temas predominantes(28). Por intermédio da análise minuciosa dos diferentes estudos, os artigos tiveram seus conteúdos agrupados em dois grandes eixos de análise temáticos, associando-se os temas predominantes que possam ter contribuído para o suicídio dos trabalhadores: aspectos internos e aspectos externos (Tabela 1).

A categoria aspectos internos abarcou aspectos emocionais e comportamentais individuais, relacionados ao trabalhador, como: a depressão $(19,3 \%)^{(18,22-26)}$; o assédio moral/bullying $(16,1 \%)^{(19-23)}$; o estresse extremo $(9,6 \%)^{(18,23-24)}$; a Síndrome de Burnout $(6,4 \%)^{(22,26)}$ e o isolamento social $(6,4 \%)^{(18-19)}$, consecutivamente. Enquanto, na categoria aspectos externos, apresentam-se fatores relevantes que não dependem do indivíduo, sendo ele exposto a fatores organizacionais e sociais, de forma subordinada, como: a ausência de lazer $(12,9 \%)^{(20-21,26-27)}$; sobrecarga profissional (horas/volume de trabalho) $(9,6 \%)^{(20-21,26)}$; acidentes no trabalho $(9,6 \%)^{(22,26-27)}$; conflitos entre a família e o trabalho $(6,4 \%)^{(26-27)}$ e a falta de autonomia no trabalho $(3,2 \%)^{(26)}$.

A associação do trabalho aos transtornos mentais é alvo de muitos questionamentos, os quais tiveram início a partir da revolução industrial(4). Com o início e a prevalência do capitalismo globalizado, a luta por direitos humanos dos trabalhadores expostos a fatores de risco sempre foi palco de grandes manifestações ${ }^{(8)}$.

Em meados de 1840, na Inglaterra, o empresário industrial alemão e teórico revolucionário Friedrich Engels escreveu a primeira obra literária voltada à saúde dos trabalhadores e condições de trabalho, a qual já trazia a temática do suicídio. A obra intitulada "A situação da classe trabalhadora na Inglaterra" é um marco inaugural na história do capitalismo e da sociedade industrial moderna. O texto de Engels apresentou uma análise observacional das péssimas condições da classe trabalhadora na Europa, das consequências inevitáveis da opressão social a qual os operários estavam expostos, assim como das doenças emergentes possivelmente decorrentes dos processos e dos locais de trabalho(29).

Como é possível observar na obra, a opressão esmagadora da indústria produtivista iniciou-se fazendo dos trabalhadores suas próprias vítimas, tanto que o autor relatou a grande frequência de suicídios na Inglaterra já naquela época(29). Novos modelos de "gestão inteligente" e a redução cada vez maior de mão de obra acarretam, para o trabalhador, um sentimento de impotência e ansiedade, aumentando, consequentemente, o nível de sujeição frente às práticas opressoras presentes no ambiente de trabalho ${ }^{(8,30)}$. 


\begin{tabular}{|c|c|c|c|c|c|c|}
\hline & Ano & País & Periódico & Autores & Título & Caracterização \\
\hline 1 & 2009 & Japão & Industrial Health & TAKADA M, et al. ${ }^{(18)}$ & $\begin{array}{l}\text { Associations between } \\
\text { lifestyle factors, working } \\
\text { environment, depressive } \\
\text { symptoms and suicidal } \\
\text { ideation: a large-scale study } \\
\text { in Japan. }\end{array}$ & $\begin{array}{l}\text { Quantitativo/Questionário } \\
\text { N: } 4.118 \text { (Homens: 68,8\%/ } \\
\text { Mulheres: } 32,2 \%)\end{array}$ \\
\hline 2 & 2011 & Brasil & $\begin{array}{l}\text { Revista Brasileira de Saúde } \\
\text { Ocupacional }\end{array}$ & $\begin{array}{l}\text { FINAZZI-SANTOS } \\
\text { MAF, SIQUEIRA MVS. } \\
\text { (19) }\end{array}$ & $\begin{array}{l}\text { Considerações sobre } \\
\text { trabalho e suicídio: um } \\
\text { estudo de caso. }\end{array}$ & $\begin{array}{l}\text { Qualitativo/Estudo de Caso } \\
\text { N: } 1 \text { (Homens: 100\%) }\end{array}$ \\
\hline 3 & 2012 & Austrália & $\begin{array}{l}\text { International journal of } \\
\text { injury control and safety } \\
\text { promotion }\end{array}$ & $\begin{array}{l}\text { ROUTLEY VH, } \\
\text { OZANNE-SMITH } \\
\text { JE. } .^{(20)}\end{array}$ & $\begin{array}{l}\text { Work-related suicide in } \\
\text { Victoria, Australia: a broad } \\
\text { perspective. }\end{array}$ & $\begin{array}{l}\text { Quantitativo/Banco de dados } \\
\mathrm{N}: 643 \text { (Homens: } 88 \% / \\
\text { Mulheres: } 12 \% \text { ) }\end{array}$ \\
\hline 4 & 2012 & $\begin{array}{l}\text { Reino } \\
\text { Unido }\end{array}$ & Crisis & PLATT B, et al. ${ }^{(21)}$ & $\begin{array}{l}\text { Suicidality in the veterinary } \\
\text { profession. }\end{array}$ & $\begin{array}{l}\text { Quantitativo/Banco de dados } \\
\mathrm{N}: 21 \text { (Homens: } 24 \% / \\
\text { Mulheres: } 76 \% \text { ) }\end{array}$ \\
\hline 5 & 2013 & Austrália & SAGE Open & 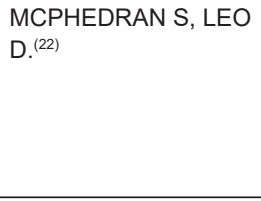 & $\begin{array}{l}\text { Suicide among miners in } \\
\text { Queensland, Australia: a } \\
\text { comparative analysis of } \\
\text { demographics, psychiatric } \\
\text { history, and stressful life } \\
\text { events. }\end{array}$ & $\begin{array}{l}\text { Quantitativo/Banco de dados } \\
\text { N: } 218 \text { (Homens: 100\%) }\end{array}$ \\
\hline 6 & 2014 & Alemanha & $\begin{array}{l}\text { Journal of Psychosomatic } \\
\text { Research }\end{array}$ & BAUMERT J, et al. ${ }^{(23)}$ & $\begin{array}{l}\text { Adverse conditions at the } \\
\text { workplace are associated } \\
\text { with increased suicide risk. }\end{array}$ & $\begin{array}{l}\text { Quantitativo/Questionário } \\
\text { N: } 28 \text { (Homens: -/Mulheres: } \\
\text {-) }\end{array}$ \\
\hline 7 & 2016 & Japão & $\begin{array}{l}\text { Journal of affective } \\
\text { disorders }\end{array}$ & WADA K, et al. ${ }^{(24)}$ & $\begin{array}{l}\text { Occupational differences } \\
\text { in suicide mortality among } \\
\text { Japanese men of working } \\
\text { age. }\end{array}$ & $\begin{array}{l}\text { Quantitativo/Banco de dados } \\
\text { N: 21(Homens: -/Mulheres: -) }\end{array}$ \\
\hline 8 & 2017 & Austrália & $\begin{array}{l}\text { BMC } \\
\text { public health }\end{array}$ & MILNER A, et al. ${ }^{(25)}$ & $\begin{array}{l}\text { Male suicide among } \\
\text { construction workers in } \\
\text { Australia: a qualitative } \\
\text { analysis of the major } \\
\text { stressors precipitating death. }\end{array}$ & $\begin{array}{l}\text { Quantitativo/Banco de dados } \\
\text { N: } 34 \text { (Homens: } 100 \% \text { ) }\end{array}$ \\
\hline 9 & 2017 & EUA & The Journal of psychology & $\begin{array}{l}\text { HOWARD M, } \\
\text { KRANNITZ M. }{ }^{(26)}\end{array}$ & $\begin{array}{l}\text { A reanalysis of occupation } \\
\text { and suicide: negative } \\
\text { perceptions of the workplace } \\
\text { linked to suicide attempts. }\end{array}$ & $\begin{array}{l}\text { Quantitativo/Banco de dados } \\
\text { N: } 2.855 \text { (Homens: } 52 \% / \\
\text { Mulheres: } 48 \% \text { ) }\end{array}$ \\
\hline 10 & 2017 & China & $\begin{array}{l}\text { Journal of Psychosomatic } \\
\text { Research }\end{array}$ & XIAO J, et al..$^{(27)}$ & $\begin{array}{l}\text { The impact of changes in } \\
\text { work stressors and coping } \\
\text { resources on the risk of new- } \\
\text { onset suicide ideation among } \\
\text { Chinese petroleum industry } \\
\text { workers. }\end{array}$ & $\begin{array}{l}\text { Quantitativo/Questionário } \\
\text { N: } 1.334 \text { (Homens: -/ } \\
\text { Mulheres: -) }\end{array}$ \\
\hline
\end{tabular}

Figura 3 - Características básicas dos estudos encontrados em função do ano de publicação. Ribeirão Preto, SP, Brasil, 2019

Tabela 1 - Análise de conteúdo dos temas apresentados nos estudos. Ribeirão Preto, SP, Brasil, 2019

\begin{tabular}{llcc}
\hline Eixos de análise & Agrupamento de temas predominantes & Frequência simples & Frequência percentual \\
\hline & Depressão & 6 & 19,3 \\
& Assédio moral/bullying & 5 & 16,1 \\
Aspectos internos & Estresse extremo & 3 & 9,6 \\
& Síndrome de esgotamento (Burnout) & 2 & 6,4 \\
& Isolamento social/individualismo & 2 & 6,4 \\
& Ausência de lazer & 4 & 12,9 \\
Sobrecarga profissional (horas/volume de & 3 & 9,6 \\
& trabalho) & 3 & 9,6 \\
& Acidente no trabalho & 2 & 6,4 \\
& Conflitos entre a família e o trabalho & 1 & 3,2 \\
\hline
\end{tabular}


Em consonância com os conceitos descritos por Engels, é possível abarcar a resultante do suicídio como um problema social decorrente da opressão e desigualdade de classes provenientes de uma organização social que tem como desígnio o capitalismo, que exige extrema produtividade e altas demandas, outorgantes para cada classe, sendo o suicídio sintoma para todas elas ${ }^{(31)}$. De fato, é possível que haja gatilhos desencadeadores entre as relações que permeiam o ambiente de trabalho, como foi possível identificar nos estudos encontrados. Nesse sentido, entende-se que o suicídio pode ser visto como uma mensagem do autor em resposta aos mecanismos que o oprimiram. Quando ocorre dentro do local de trabalho, podem-se indicar uma defraudação das relações interpessoais e o enfraquecimento de solidariedade nesse contexto. Já fora do local de trabalho, é possível interpretar tal ato como uma canalização da sujeição ao sofrimento no cenário trabalhista para fora da cena a fim de preservar os resquícios de coletividade defensiva existentes ${ }^{(32)}$.

Porém, a relação de causalidade, mesmo diante de muitas outras causas, que fundamentam o vínculo entre saúde/doença mental e trabalho, não daria conta de determinar todas as possíveis manifestações humanas. Assim, é impossível determinar uma única causa de desfecho nessas situações extremas de estresse e empobrecimento psicológico(26-27,33). Portanto, o conceito de saúde mental não é necessariamente a ausência aparente de transtornos psíquicos(33).

É necessário levar em conta as dimensões subjetivas dos mecanismos e comportamentos psíquicos de adoecimento que, certamente, influenciarão a relação do homem com o seu ambiente de trabalho(18,20-21,32-33). Entretanto, esses agravos relatados nos estudos encontrados, como o bullying e o estresse, podem levar ao isolamento social, desencadeando a depressão e, em casos extremos, os pensamentos suicidas(18-19,22-26), que são ainda pouco investigados e/ou raramente registrados ${ }^{(34)}$.

Nesse sentido, a vigilância em saúde do trabalhador torna-se de extrema importância para a intervenção, sendo ela capaz de, primeiramente, dar espaço de fala à voz interna desse sujeito sem que ele seja coagido, com medo de retaliação ou julgamentos ${ }^{(33-35)}$, identificando esses gatilhos desencadeadores supracitados, que estão inteiramente associados aos aspectos externos aos quais o indivíduo está exposto no ambiente de trabalho. No Brasil, são objetivos dos órgãos responsáveis à saúde do trabalhador, como os CERESTs e a RENAST, a assistência e a vigilância desse público por meio da atuação, monitoria, prevenção, notificação e enfrentamento estratégico dos problemas de saúde coletiva, como as mortes, os acidentes e as doenças relacionados com o trabalho( ${ }^{(36)}$. Encontrou-se, nos artigos dessa RI, desde a privação de horas para lazer, com demandas excessivas de carga horária, até a perda de saúde, identidade e relações sociais, mecanismos extremamente importantes para a manutenção da saúde mental(20-22,26-27).

A depressão, doença (fator) apontada nos resultados(18,22-26), já é considerada o grande mal deste século(37). Segundo a OMS, até 2020, a depressão estará colocada em segundo lugar entre as principais causas de incapacidade para o trabalho em todo o mundo. Estima-se que hoje, no Brasil, haja 17 milhões de pessoas sofrendo com a doença, sendo 121 milhões de pessoas em todo mundo(38).

Outro dado eminente e muito comentado nos estudos é o assédio moral/bullying no trabalho(20-24), que se caracteriza pela exposição de trabalhadores a situações humilhantes e constrangedoras, com diferenças hierárquicas, negativas e desumanas de longa duração no ambiente de trabalho(39). Isso pode levar o trabalhador à ansiedade intensa e, consecutivamente, ao desencadeamento de doenças como a depressão e, ainda, às causas piores quando os ataques e constrangimentos são contínuos, culminando com a demissão(40).

Vale ressaltar que violências morais dentro do ambiente de trabalho possuem barreiras invisíveis de difícil detecção pelas esquipes de saúde e também pela própria vítima, sendo, na maioria das vezes, não materiais e intangíveis, abrangendo os campos da subjetividade e clareza de fatos, os quais podem favorecer uma linha tênue entre os deveres e a dominação, assim como entre a função e a servidão. Como resultantes dessas exposições, sinais e sintomas de estresse pós-traumático e Síndrome de Burnout podem ser diagnosticados, assim como distúrbios fisiológicos (cardíacos, endócrinos e digestivos), alcoolismo, drogadição e até o suicídio em consumação(41).

Dentre os aspectos internos, os riscos de estresse extremo pós-traumático e da síndrome de esgotamento (Burnout) são potencializados quando associados ao trabalho de modo lento e, na maioria das vezes, imperceptível(22,26). A doença acentua-se em razão do aumento das pressões do dia a dia, em que a competição incessante pelas metas pessoais e em tempo acelera o ritmo de trabalho, tornando-o extremamente cansativo e elevando exponencialmente os níveis de estresse e, também, mediante as ameaças de mudanças na jornada de trabalho e na ameaça de crise econômica(42).

CERESTs regionais e estaduais possuem papel importante enquanto facilitadores de ações para a identificação e o reconhecimento dos trabalhadores expostos a riscos, que permanecem, muitas vezes, invisíveis e inacessíveis ${ }^{(36)}$. Somadas à Política Nacional de Atenção Básica (PNAB) (responsável pela formação 
de equipes de saúde: médicos, enfermeiros e auxiliares) e aos Núcleos de Apoio à Saúde da Família (NASF) (especializados na promoção da saúde da população na atenção básica) ${ }^{(43)}$, a articulação de trabalho e a troca de informações entre esses departamentos citados provavelmente ajudariam na detecção desses indivíduos em situação de perigo no trabalho, prevenindo extremos e promovendo a saúde por meio de diretrizes, estratégias e ações que possam ir ao encontro dos problemas detectados que permeiam determinado grupo. Esse olhar prévio é o mais criterioso facilitador da vigilância e promoção da saúde, buscando identificar momentos oportunos de intervenção(9).

Ao pensar nisso, há muito, propôs-se uma classificação das doenças relacionadas ao trabalho, denominada "Classificação de Schilling". Tal exemplo de ferramenta já validada pode ser útil para a determinação dos fatores precipitantes, a observação e o relato de exposições a agentes estressores psicológicos e/ou físicos presentes no ambiente físico de trabalho(44-45).

Debruça-se sobre um agravo importante quanto aos transtornos mentais associados ao trabalho: não são catalogados, na Classificação Estatística Internacional de Doenças e Problemas Relacionados com a Saúde (CID), os casos de ideação suicida, tentativa de suicídio e/ou o suicídio(5). Isso posto, é possível que a falta da classificação desses sinais e sintomas dificulte sua identificação efetiva, não permitindo a realização de diagnósticos prévios da ocorrência desses eventos na população, os quais poderiam fornecer recursos para explicações causais desses agravos.

Como supracitado, a associação de transtornos mentais com o trabalho é alvo de estudos que recomendam a proteção especial e o auxílio aos trabalhadores expostos e/ou afetados aos riscos laborais, com direitos reais a afastamentos, benefícios da Previdência, medidas preventivas, dentre outros. Entretanto, no contexto de vigilância em saúde do trabalhador, os nexos ocupacionais desses agravos precisam ser melhor investigados a fim de livrar-se de objetos de incertezas e conflitos de interesses permanentes entre a instituição e o trabalhador ${ }^{(34,41)}$

A exemplo do SUS, transtornos mentais associados ao trabalho são de notificação compulsória no Sistema de Informação de Agravos de Notificação (SINAN), no qual sua divulgação de conhecimento é priorizada pela OMS desde 2019. Porém, a identificação por meio dos registros desses casos no SINAN ainda é pouco registrada, não sendo possível explorar profundamente os dados ${ }^{(5)}$.

Assim, cabe analisar, discutir e identificar esses vieses de notificação, possivelmente encontrados no nexo causal entre o ambiente e o empobrecimento mental que leva o trabalhador a consumar o suicídio ${ }^{(34)}$.
Em uma perspectiva de caracterização do fato ocorrido, associada à notificação compulsória prévia, colaborarse-ia para a identificação de acordo com cada realidade epidemiológica e determinada área geográfica, suscitando, assim, medidas de vigilância e prevenção mais eficazes contra esses indicadores.

Outro ponto destacável é sobre o investimento em campanhas educativas para a promoção da saúde mental e prevenção do suicídio. Assim como outros assuntos referentes à temática, a falta de uniformização e incentivo de campanhas constantes, que vão ao encontro desse público carente de esclarecimentos e diálogo, facilitaria a discussão do assunto em períodos contínuos e não apenas em campanhas pontuais, como "Setembro Amarelo", realizada apenas no mês de setembro(46). Esse investimento pode minimizar a falta de sensibilidade e o preconceito oriundos de algumas das partes relacionadas quanto à expressão confessa da ideação suicida, marcada por atitudes negativas e condenatórias ao emissor de comportamento suicida, que interfere penosamente na qualidade do cuidado prestado ao mesmo ${ }^{(47)}$. Dessa forma, geram-se a falta de notificação e, principalmente, a falta do tratamento, já que o indivíduo entende que falar desse assunto é uma atitude condenatória e censurável(48).

No campo das ações preventivas, emerge a necessidade de contratação e treinamento de equipe de saúde especializada para detecção e diagnósticos psicossociais contínuos no meio ambiente do trabalho. As medidas preventivas podem ser de origem primária (resolução imediata de conflito), secundária (detecção da origem) e terciária (implantação de ação para erradicação), incluindo indicadores de desempenho, com a elaboração de protocolos de ação e melhorias no clima organizacional da instituição, sob uma cultura de ciclo de gestão organizacional (Ciclo PDCA: Planejar, Fazer, Checar e Executar), com a elaboração de protocolos de ação dentro da empresa com a presença e a participação dos trabalhadores ${ }^{(41)}$

Por fim, o preconceito associado à falta de espaço para a discussão é aspecto subjacente, que pode fortalecer a ideação suicida. Portanto, acredita-se que, ao compartilhar seus medos e angústias, o indivíduo torna-se capaz de autogerenciar sentimentos e acrescentar significados à experiência da vida, a ressignificando(49).

Esta revisão integrativa da literatura apresenta, como limitação, a não abrangência da pesquisa em todas as bases de dados bibliográficos disponíveis, embora tenha havido o cuidado de abranger as principais que permeassem a temática central do estudo. Outra limitação é a falta de dados de gênero em três $\operatorname{artigos}^{(23-24,27)}$. A categorização simplificada dos aspectos na análise dos dados pode implicar a perda de clareza 
aprofundada na discussão de cada estudo selecionado, embora reflita a complexidade epistêmica e metodológica do campo proposto. Apesar dessas limitações, destaca-se a convergência do escopo temático nas discussões entre os estudos selecionados, bem como a diversidade de métodos analisados. Analisar artigos em três idiomas é mais um mérito desta revisão. Ressalta-se, ainda, o ineditismo de correlacionar as grandes áreas de estudo: vigilância em saúde do trabalhador e suícidio, campos ainda pouco exploados em estudos atuais.

\section{Conclusão}

Ao partir de categorias-chave enfatizadas no campo da vigilância em saúde do trabalhador, esta revisão trouxe as possíveis relações que permeiam a ideação suicida e o suicídio, relacionadas aos aspectos internos e externos do trabalho, que podem ser percebidas e melhoradas por meio do papel da equipe de Enfermagem, principalmente do trabalho.

Constatou-se que políticas e programas públicos devem ir ao encontro do aprofundamento deste olhar, não apenas ao tratar a doença, mas, também, ao fiscalizar e melhorar o ambiente e as condições de trabalho aos quais estes indivíduos estão expostos, garantindo, aos mesmos, o zelo pela integridade e pela saúde, a qual, no seu conceito mais amplo, não pressupõe apenas a ausência de doenças.

Mostra-se que, mesmo com a ampla fundamentação das políticas brasileiras de saúde do trabalhador, diversos são os fatores que podem levar o trabalhador à ideação suicida e, quando não assistido, ao suicídio. É dever de toda instituição empregadora (pública ou privada) garantir a vigilância e prevenção à saúde a fim de promover ambientes e condições de trabalho seguros e saudáveis que protejam o trabalhador.

Destaca-se, ainda, a necessidade de direcionar campanhas educativas contínuas de prevenção ao suicídio e promoção à saúde no meio ambiente do trabalho de acordo com cada grupo, operando a responsabilidade social, a começar nas relações interpessoais humanizadas e em seus próprios ambientes institucionais internos.

Os achados deste estudo mostram a necessidade de suscitar e desenvolver outras pesquisas sobre a temática ainda pouco discutida na literatura, explorando aspectos igualmente relevantes que permeiem a vigilância em saúde, a saúde do trabalhador e o suicídio.

\section{Agradecimentos}

À Coordenação de Educação a Distância da Escola Nacional de Saúde Pública Sergio Arouca - EAD/ENSP, da Fundação Oswaldo Cruz - FIOCRUZ, e, em especial, à Dra. Marcia Schaid e Dra. Márcia Tiveron.

\section{Referências}

1. Pan American Health Organization (PAHO). World Health Organization (WHO). Fact sheet: Suicide in Brazil [Internet]. Brazil; 2018 [cited Aug 9 2019]. Available from: https://www.paho.org/bra/index. php?option $=$ com_content\&view $=$ article\&id $=5671:$ fo Iha-informativa-suicidio\&Itemid=839.

2. Ferreira GS, Fajardo AP, Mello, ED. Possibilities of addressing suicide in the Family Health Strategy. Physis. 2019;29(4):e290413. doi: 10.1590/ S0103-73312019290413

3. Emergency Nurses Association. Clinical practice guideline: suicide risk assessment. [Internet] Schaumburg; 2018 [cited Dec 20 2019]. Available from: https://www.intljourtranur.com/action/showPdf?p $\mathrm{ii}=\mathrm{S} 0099-1767 \% 2818 \% 2930350-7$.

4. Costa DM, Lacaz FAC, Jackson Fo JM, Vilela RAG. Worker's Health within the Brazilian Unified Health System: challenges for a public policy. Rev Bras Saúde Ocup. 2013;38(127):11-30. doi: 10.1590/ S0303-76572013000100003

5. Almeida MMC. Colaborador da Vigilância dos Agravos à Saúde do Trabalhador. Boletim Epidemiológico: Transtornos Mentais Relacionados ao Trabalho no Brasil, 2006-2017. [Internet] Health Surveillance Secretariat, Brazil; 2019 [cited Dec 15 2019]. Available from: https:// www.analisepoliticaemsaude.org/oaps/documentos/ noticias/ccvisat-bol-transtmentaisfinal-260419.

6. Matthews LR, Bohle P, Quinlan M, Rawlings-Way $O$. Traumatic Death at Work: Consequences for Surviving Families. Int J Health Serv. 2012;42(4):647-66. doi: 10.2190/HS.42.4.e

7. Ministério da Casa Civil (BR). Decreto n 7.602, de 07 de novembro de 2011. Dispõe sobre a Política Nacional de Segurança e Saúde no Trabalho - PNSST. Diário Oficial da República Federativa do Brasil, Brasília, DF, 8 nov 2011. [Acesso 12 dez 2019]. Disponível em: http:// www. planalto.gov.br/.

8. Pintor E, Garbin AC. Work-related violence registries and worker's health surveillance: breaking invisibility. Rev Bras Saúde Ocup. 2019;44:e20. doi: 10.1590/2317-6369000006918

9. Ministério da Casa Civil (BR). Portaria no 1.823, de 23 de agosto de 2012. Institui a Política Nacional de Saúde do Trabalhador e da Trabalhadora - PNSTT. Diário Oficial da República Federativa do Brasil, Brasília, DF, 23 ago. 2012. . [Acesso 10 jul 2019]. Disponível em: http://bvsms.saude.gov.br/bvs/saudelegis/gm/2012/ prt1823_23_08_2012.html

10. Amorim LDA, Silva TL, Faria HPD, Machado JMH, Dias EC. Worker's Surveillance in the Primary Care: learning with Family Health team of João Pessoa, Paraíba, Brazil. Ciênc Saúde Coletiva. 2017;22(10):3403-13. doi: 10.1590/1413-812320172210.17532017

11. Paula CC, Padoin SMM, Galvão CM. Revisão integrativa como ferramenta para tomada de decisão 
na prática de saúde. In: Lacerda MR, Costenaro RGS, editores. Metodologias da pesquisa para a enfermagem e saúde: da teoria à prática. Porto Alegre: Moriá; 2015. p. 51-76.

12. Corsi CAC. Estratégias educativas utilizadas na educação básica para conscientização dos alunos sobre a temática doação/transplante de órgãos e tecidos humanos: revisão integrativa da literatura [dissertação]. Ribeirão Preto (SP): Escola de Enfermagem de Ribeirão Preto, Universidade de São Paulo; 2018.

13. Carrara GLR. A utilização de instrumentos para avaliação da liderança nos serviços de saúde e enfermagem [dissertação]. Ribeirão Preto (SP): Escola de Enfermagem de Ribeirão Preto, Universidade de São Paulo; 2016.

14. Mendes KD, Silveira RC, Galvão CM. Integrative literature review: a research method to incorporate evidence in health care and nursing. Texto Contexto Enferm. 2008;17(4):758-64. doi: 10.1590/ S0104-07072008000400018

15. Joanna Briggs Institute. 'Joanna Briggs Institute Reviewers Manual: 2014' Edition. [Internet]. Adelaide: Joanna Briggs Institute; 2014. Available from: http://joannabriggs.org/assets/docs/sumari/ ReviewersManual-2014.pdf.

16. Whittemore $R, K$ nafl $K$. The integrative review: updated methodology. J Adv Nurs. 2005;52(5):546-53. doi: 10.1111/j.1365-2648.2005.03621.x

17. Shamseer $L$, Moher $D$, Clarke M, Ghersi D, Liberati $A$, Petticrew $M$, et al. Preferred reporting items for systematic review and meta-analysis protocols (PRISMA-P) 2015: elaboration and explanation. BMJ. 2015;349:1-25. doi: 10.1136/bmj.g7647

18. Takada M, Suzuki A, Shima S, Inoue K, Kazukawa S, Hojoh, M. Associations between lifestyle factors, working environment, depressive symptoms and suicidal ideation: a large-scale study in Japan. Ind Health. 2009;47(6):649-55. doi: 10.2486/indhealth.47.649

19. Finazzi-Santos MA, Siqueira MVS. Considerations on work and suicide: a case study. Rev Bras Saúde Ocup. 2011;36(123):71-83. doi: 10.1590/ S0303-76572011000100007

20. Routley VH, Ozanne-Smith JE. Work-related suicide in Victoria, Australia: a broad perspective. Int J Inj Contr Saf Promot. 2012;19(2):131-4. doi: 10.1080/17457300.2011.635209

21. Platt B, Hawton K, Simkin S, Dean R, Mellanby RJ. Suicidality in the veterinary profession: interview study of veterinarians with a history of suicidal ideation or behavior. Crisis. 2012;33(5):280-9. doi: 10.1027/0227-5910/a000143

22. McPhedran S, De Leo D. Suicide among miners in Queensland, Australia: a comparative analysis of demographics, psychiatric history, and stressful life events. Sage Open. 2013;3(4). doi: $10.1177 \% 2 F 2158244013511262$
23. Baumert J, Schneider B, Lukaschek K, Emeny RT, Meisinger $C$, Erazo $N$, et al. Adverse conditions at the workplace are associated with increased suicide risk. J Psychiatr Res. 2014;57:90-95. doi: 10.1016/j. jpsychires.2014.06.007

24. Wada K, Eguchi H, Prieto-Merino D, Smith DR. Occupational differences in suicide mortality among Japanese men of working age. J Affect Disord. 2016;190:316-21. doi: 10.1016/j.jad.2015.10.032

25. Milner A, Maheen $H$, Currier D, LaMontagne AD. Male suicide among construction workers in Australia: a qualitative analysis of the major stressors precipitating death. BMC Public Health. 2017;17(1):584. doi: 10.1186/s12889-017-4500-8

26. Howard M, Krannitz M. A reanalysis of occupation and suicide: Negative perceptions of the workplace linked to suicide attempts. J Psychol. 2017;151(8):767788. doi: $10.1080 / 00223980.2017 .1393378$

27. Xiao J, Guan S, Ge H, Tao N, Zhang Y, Jiang Y, et al. The impact of changes in work stressors and coping resources on the risk of new-onset suicide ideation among Chinese petroleum industry workers. J Psychiatr Res. 2017;88:1-8. doi: 10.1016/j.jpsychires. 2016.12.014

28. Cortez PA, Veiga HMS, Gomide APA, Souza MVR. Suicide at work: review of Brazilian literature in Psychology. rPOT. 2019;19(1):523-531. doi: 10.17652/ rpot/2019.1.14480

29. Engels F. A situação da classe trabalhadora na Inglaterra. 2th ed. São Paulo: Boitempo 2010. p. 150-5. 30. Venco S, Barreto $M$. The social meaning of suicide at work. REA. [Internet]. 2010; Apr. 26 [cited 2020 Jul 8]; 9(108): 1. Available from: http://www.periodicos.uem. br/ojs/index.php/EspacoAcademico/article/view/10032 31. De Campos LJ, Alquatti R, Garbin SR, Pereira I. Work and suicide: an ultimate resistance gesture. Est Pesqui Psicol. 2016;16(1):86-103. doi: https://doi. org/10.12957/epp.2016.24832

32. Dejours C, Bègue F. Suicídio e trabalho: o que fazer?. Brasília: Paralelo. 2010;15: 128.

33. Jacques MG. The causal nexus on mental health/ illness related to work: a new demand for psychology. Psicol Soc. 2007;19(spe):112-9. doi: 10.1590/ S0102-71822007000400015

34. Soares A, Oliveira JA. Bullying at work. Rev Bras Saúde Ocup. [Internet]. 2012 Dec [cited 2020 Jul 21]; 37( 126 ): 195-202. Available from: http://www. scielo.br/scielo.php?script=sci_arttext\&pid=S0303$76572012000200002 \& \mathrm{lng}=$ en. doi:10.1590/ S0303-76572012000200002.

35. Pan American Health Organization (PAHO). World Health Organization (WHO). Work-related diseases: manual of procedures for health services. Brazil; 2001 [cited Aug 19 2019]. Available from: https://www.paho.org/bra/index.php?option=com_ docman\&view =download\&alias $=207$-doencasrelacionadas-ao-trabalho-manual-procedimentos-para- 
os-servicos-saude-7\&category_slug=saude-e-ambiente707\&Itemid $=965$

36. Ministério da Saúde (BR). Portaria GM/MS n 2.437, de 7 de dezembro de 2005. Dispõe sobre a ampliação e o fortalecimento da Rede Nacional de Atenção Integral à Saúde do Trabalhador - RENAST no Sistema Único de Saúde - SUS e dá outras providências. Diário Oficial da República Federativa do Brasil, Brasília, DF, 7 dez. 2005. [Acesso 29 set 2019]. Disponível em: https://www. saude.mg.gov.br/images/documentos/portaria2437.pdf 37. Rocha ME, Freire KP, Dos Reis WPD, Vieira LTQ, De Sousa LM. Factors that occur the index of depressive and anxiety disorders in nursing professionals: a bibliographic review. Braz J Dev. 2020;6(2):9288-305. doi: $10.34117 /$ bjdv6n2-296

38. Lelis KCG, Brito RVNE, Pinho S, Pinho L. Symptoms of depression, anxiety and drug use among university students. Rev Portuguesa Enferm Saúde Mental. [Internet]. 2020 [cited July 8 2020]; (23):9-14. doi: 10.19131/rpesm.0267

39. Figueiredo AM Júnior, Pereira JS, Cardoso JAC, Santa Brígida GV, Frazão J M, Fernandes CS, et al. Scientific production about bullying in nursing work relationships in Brazil. Rev Esc Enferm USP. 2020;3:e2742. doi: 10.25248/reaenf.e2742.2020

40. Timenetsky KT, Castro CA, Eid RC, CarnieliCazati D. Prevalence of burnout syndrome among physiotherapist working in intensive care units and step down unit. Intensive Care Med Exp. 2015;3(suppl 1):A722. doi: 10.1186/2197-425X-3-S1-A722

41. Barreto MMS. Assédio moral: trabalho, doenças e morte. In: Lima CQB, Oliveira JA, Maeno M. Seminário compreendendo o assédio moral no ambiente de trabalho. São Paulo: Fundacentro; 2013. p. 13-26. Disponível em: http://www.assediomoral.ufsc.br/files/2013/03/ Seminario-Combate-AMT-Fundacentro-2013.pdf 42. Moraes SM, Magrini DF, Zanetti ACG, Santos MA, Vedana KGG. Attitudes and associated factors related to suicide among nursing undergraduates. Acta Paul Enferm. 2016;29(6):643-49. doi: 10.1590/1982-0194201600090

43. Ministério da Saúde (BR). Portaria n 2.436, de 21 de setembro de 2017. Aprova a Política Nacional de Atenção Básica, estabelecendo a revisão de diretrizes para a organização da Atenção Básica, no âmbito do Sistema Único de Saúde (SUS). Diário Oficial da República Federativa do Brasil, Brasília, DF, 21 set. 2017. [Acesso 20 jul 2020]. Disponível em: https://bvsms.saude.gov. br/bvs/saudelegis/gm/2017/prt2436_22_09_2017.html
44. Schilling RSF. More effective prevention in occupational health practice? J Soc Occup Med. 1984;34(3):71-9. doi: 10.1093/occmed/34.3.71

45. Dias EC. Doenças relacionadas ao trabalho: manual de procedimentos para os serviços de saúde. Brasília: Ministério da Saúde do Brasil; 2001. p. 580. Disponível em: http://bvsms.saude.gov.br/bvs/publicacoes/ doencas_relacionadas_trabalho_manual_procedimentos. pdf

46. Oliveira MEC, Gomes KAL, Nóbrega WFS, Gusmão ECR, dos Santos RD, Franklin RG. Temporal suicide series in Brazil: what changed after the "Setembro Amarelo"?. REAS. 2020;(48):e3191. doi: 10.25248/ reas.e3191.2020

47. Alves R. Conversas com quem gosta de ensinar. 10th ed. Campinas: Papirus; 2008. p. 15-7.

48. Manyalich M, Mestres CA, Ballesté C, Páez G, Valero R, Gómez MP. Organ procurement: Spanish transplant procurement management. Asian Cardiovasc Thorac Ann. 2011;19(3-4):268-78. doi: $10.1177 / 0218492311411590$

49. Paiva LE. A arte de falar da morte para crianças. Aparecida: Ideias \& Letras; 2011. p. 35-9.

\section{Contribuição dos autores}

Concepção e planejamento do estudo: Carlos Alexandre Curylofo Corsi, Alan Vinicius Assunção Luiz, Álefe Saloum Cintra, Natássia Condilo Pitta e Talita Silva Queiroz. Obtenção dos dados: Carlos Alexandre Curylofo Corsi, Talita Silva Queiroz e Alan Vinicius Assunção Luiz. Análise e interpretação dos dados: Carlos Alexandre Curylofo Corsi, Alan Vinicius Assunção Luiz, Álefe Saloum Cintra, Natássia Condilo Pitta e Talita Silva Queiroz. Análise estatística: Carlos Alexandre Curylofo Corsi, Alan Vinicius Assunção Luiz, Álefe Saloum Cintra e Natássia Condilo Pitta. Redação do manuscrito: Carlos Alexandre Curylofo Corsi, Alan Vinicius Assunção Luiz, Álefe Saloum Cintra e Natássia Condilo Pitta. Revisão crítica do manuscrito: Milena Flória-Santos e Ana Cláudia da Silva Paschoal.

Todos os autores aprovaram a versão final do texto.

Conflito de interesse: os autores declararam que não há conflito de interesse.
Copyright ( 2020 SMAD, Rev. Eletrônica Saúde Mental Álcool Drog. Este é um artigo de acesso aberto distribuído sob os termos da Licença Creative Commons CC BY-NC.

Esta licença permite que outros remixem, adaptem e criem a partir do eu trabalho para fins não comerciais, e embora os novos trabalhos tenham de lhe atribuir o devido crédito e não possam ser usados para fins comerciais, os usuários não têm de licenciar esses trabalhos derivados sob os mesmos termos. 